\title{
Use of Robotic Surgery for Teaching Anatomy, Clinical Anatomy and Surgical Anatomy to Medical Students in the Operating Room
}

\section{Canda $\mathrm{AE}^{*}$}

Associate Professor of Urology, Deputy Dean, School of Medicine, Ankara Yildirim Beyazit University, Ankara Ataturk Training and Research Hospital, Department of Urology, Ankara, Turkey

\section{Introduction}

Teaching human anatomy training is a very important part of medical training. Depending on the medical school, anatomy lectures might start on first or second year of the medical school. Understanding anatomy and clinical anatomy is very important for medical students in order to learn the diseases.

Robotic surgery is increasingly being used in the world. In urology, robotic surgery is most frequently applied in prostate, urinary bladder, kidney and adrenal gland diseases. Surgical robot gives the advantages of precise, gentle and quick tissue handling with three-dimensional (3D) magnified image capability, higher grades of wristed hand movements and decreased hand tremor. Therefore, all details of the anatomical structures could be demonstrated nicely in robotic surgery with magnified vision. In our robotic surgery operating room, we have a large sized high-definition (HD) monitor in addition to a large sized $3 \mathrm{D}$ monitor that creates a $3 \mathrm{D}$ view if the surgical procedure is watched by putting on $3 \mathrm{D}$ eyeglasses. We use both of the monitors for training purposes for colleagues who visit our operating room in order to watch the robotic urological cases.

Medical students are very much interested in high technology procedures and robotic surgery has always been a very attractive type of surgery for them. At Ankara Yildirim Beyazit University, medical students have requested to watch robotic surgical procedures in the operating room. They we allowed to observe and watch the cases (two or three medical students per case). Their willing to learn has prompted us to explain the anatomy, clinical anatomy and even surgical anatomy during performing robotic urological procedures in the operating room. In addition, we also give information depending on the level of the medical students about the urological diseases including symptoms, diagnosis and management. Likewise, we give detailed answers to the questions asked by the medical students such as why we do robotic surgery, what are the outcomes and how is the postoperative patient follow-up. After removing the specimen out of the patient's body at the end of the robotic surgery, we examine the removed organ (i.e. prostate, kidney, adrenal gland) with the medical students. Due to my personal observation, medical students have always been very much curious and exited to watch the robotic urological procedures on large screens with $3 \mathrm{D}$ vision and examine the removed organ with their hands after putting on surgical gloves. Robotic surgery could be used as a very attractive teaching modality for teaching anatomy, clinical anatomy, surgical anatomy and urological diseases not only to residents but also to medical students. A more efficient and structured teaching program could be developed by including an anatomist in the operating room who could give much more detailed information about human anatomy to medical students during we perform robotic surgical procedures that could lead to a long lasting and enjoyable way of medical training.

*Corresponding author: Canda AE, Associate Professor of Urology, Deputy Dean, School of Medicine, Ankara Yildirim Beyazit University, Ankara Ataturk Training and Research Hospital, Department of Urology, Ankara, Turkey, Tel: 90 506763 5466; E-mail: erdemcanda@yahoo.com

Received February 07, 2015; Accepted February 10, 2015; Published February 17, 2015

Citation: Canda AE (2015) Use of Robotic Surgery for Teaching Anatomy, Clinical Anatomy and Surgical Anatomy to Medical Students in the Operating Room. Adv Robot Autom 4: e125. doi:10.4172/2168-9695.1000e125

Copyright: (c) 2015 Canda AE. This is an open-access article distributed under the terms of the Creative Commons Attribution License, which permits unrestricted use, distribution, and reproduction in any medium, provided the original author and source are credited. 